\title{
Safe and effective management of Tracheostomy in COVID-19 patients
}

\author{
Hongjun Xiao, $\mathrm{MD}^{1}$ \\ ${ }^{1}$ Union Hospital, Tongji Medical College
}

May 5, 2020

\begin{abstract}
Background: An increasing number of COVID-19 patients worldwide will probably need tracheostomy in an emergency or at the recovering stage of COVID-19. We explored the safe and effective management of tracheostomy in COVID-19 patients, to benefit patients and protect healthcare workers at the same time.

Methods: We retrospectively analyzed 11 hospitalized COVID-19 patients undergoing tracheostomy. Clinical features of patients, ventilator withdrawal after tracheostomy, surgical complications and nosocomial infection of the healthcare workers associated with the tracheostomy were analyzed.

Results: All the tracheostomy of 11 cases (100\%) were performed successfully, including percutaneous tracheostomy of 6 cases $(54.5 \%)$ and conventional open tracheostomy of 5 cases $(45.5 \%)$. No severe postoperative complications occurred, and no healthcare workers associated with the tracheostomy are confirmed to be infected by SARS-CoV-2.

Conclusion: Comprehensive evaluation before tracheostomy, optimized procedures during tracheostomy, and special care after tracheostomy can make the tracheostomy safe and beneficial in COVID-19 patients.

Xiaomeng Zhang ${ }^{1}$, MD; Qiling Huang ${ }^{1}$, MD; Xun Niu, MD ${ }^{1}$; Tao Zhou ${ }^{1}$, MD; Zhen Xie ${ }^{1}$, MD; Yi Zhong ${ }^{1}$, $\mathrm{MD}$; Hongjun Xiao ${ }^{1}, \mathrm{MD}$

${ }^{1}$ Department of Otorhinolaryngology, Union Hospital, Tongji Medical College, Huazhong University of Science and Technology, Wuhan, China
\end{abstract}

\section{Correspondence:}

Hongjun Xiao, Department of Otorhinolaryngology, Union Hospital, Tongji Medical College, Huazhong University of Science and Technology, 1277 Jiefang Ave, 430022, Wuhan, China.

Email: xhjent@163.com

Yi Zhong, Department of Otorhinolaryngology, Union Hospital, Tongji Medical College, Huazhong University of Science and Technology, 1277 Jiefang Ave, 430022, Wuhan, China.

Email: zyzy0411@163.com

These authors contributed equally: Xiaomeng Zhang and Qiling Huang.

\section{Abstract}


Background: An increasing number of COVID-19 patients worldwide will probably need tracheostomy in an emergency or at the recovering stage of COVID-19. We explored the safe and effective management of tracheostomy in COVID-19 patients, to benefit patients and protect healthcare workers at the same time.

Methods: We retrospectively analyzed 11 hospitalized COVID-19 patients undergoing tracheostomy. Clinical features of patients, ventilator withdrawal after tracheostomy, surgical complications and nosocomial infection of the healthcare workers associated with the tracheostomy were analyzed.

Results: All the tracheostomy of 11 cases (100\%) were performed successfully, including percutaneous tracheostomy of 6 cases (54.5\%) and conventional open tracheostomy of 5 cases (45.5\%). No severe postoperative complications occurred, and no healthcare workers associated with the tracheostomy are confirmed to be infected by SARS-CoV-2.

Conclusion: Comprehensive evaluation before tracheostomy, optimized procedures during tracheostomy, and special care after tracheostomy can make the tracheostomy safe and beneficial in COVID-19 patients.

\section{INTRODUCTION}

Beginning in December 2019, a novel coronavirus (SARS-CoV-2), has caused an international outbreak of acute respiratory illness termed COVID-191-3. Up to April 26, 2020, more than 2,943,000 infections and 204,000 deaths are reported in worldwide. So far, there are no specific therapeutic agents for the treatment of COVID-19. For some hospitalized patients with severe COVID-19, endotracheal intubation and mechanical ventilation is a common and indispensable life-saving treatment ${ }^{4-5}$.

In COVID-19 patients with long-term endotracheal intubation and mechanical ventilation, tracheotomy may be considered ${ }^{6-7}$. However, many clinicians think tracheotomy should be avoided if at all possible, because the risk arises not only during the procedure but also during the process of the care for the patients afterwards. Therefore, tracheotomy has become known as one of the most high-risk surgical procedures in patients with Covid-19. Usually percutaneous dilational tracheotomy is preferred to be used, which is a minimally invasive method that can be performed at the bedside ${ }^{8}$. But some patients with unfavorable neck anatomy, including short neck, enlarged thyroid, neck cicatricial contracture and so on, are not considered to be suitable for percutaneous tracheotomy. In these circumstances, conventional tracheotomy is an inevitable choice for surgeons who perform tracheotomy. During conventional open tracheotomy, the opening of the airway is more prone to cause secretion spatter which is aerosol-generating and may contribute to nosocomial spread of viruses from patients to operating room personnel. The SARS-CoV-2 virus from patients can remain viable and infectious in aerosols for hours and on surfaces up to days ${ }^{9}$, paving the way for nosocomial spread. Therefore, whether the COVID-19 patients need to undergo tracheotomy and how to avoid nosocomial spread during tracheostomy or after tracheostomy is crucial to benefit the patients and prevent healthcare workers from the risk of transmission of coronavirus.

In the past three months, our team in Wuhan Union Hospital (West Branch), which was a designated key hospital for severe Covid-19 patients in Wuhan, China, developed experience in treating Covid-19 patients, including various drug-treatments and tracheostomy. The number of patients with laboratory-confirmed SARS-CoV-2 infection continues to grow as many countries involved. In the following months, an increasing number of Covid-19 patients worldwide will probably need long-term endotracheal intubation and possibly even tracheotomy. Here, we report the clinical characteristics of COVID-19 patients with tracheostomy and management of tracheostomy in COVID-19 patients in our hospitals, which are located in the epicenter of China.

\section{PATIENTS AND METHODS}

\subsection{Study design}


This retrospective, observational study was done at the designated special care center for severe COVID19 patients of Union Hospital (West Branch) of Huazhong University of Science and Technology (Wuhan, China). This center is one of the designated hospitals assigned by the government to treat patients with COVID-19. We retrospectively analyzed consecutive hospitalized patients from January 23, 2020, to April 6, 2020, who had been diagnosed as having COVID-19. A case of COVID-19 was confirmed by a positive result of SARS-CoV-2 infection on highthroughput sequencing or real-time reverse-transcription polymerase chain reaction analysis of throat swab specimens. A total of 1361 hospitalized patients with laboratory confirmation of SARS-CoV-2 infection were identified in this center. Of these, 11 medical records were available for review. Cases were screened for those performed by a tracheostomy, including percutaneous tracheostomy performed by intensive care doctors, and conventional open tracheostomy performed by otolaryngologists from our team. Indications for surgery, surgical complications and adjuvant therapy were identified from the patient's clinical records. This study was approved and written informed consent was waived by the Ethics Committee of Union hospital, owing to the rapid emergence of the disease and the urgent need to collect data. At the time of treatment, extensive discussion was conducted with the patient or a relative for patients who could not give consent, describing the risks and benefits of surgery.

\subsection{Data Collection}

We reviewed electronic medical records, nursing records, and laboratory findings for all COVID-19 patients undergoing tracheotomy and collected data. The baseline characteristics contained demographic, comorbidities, endotracheal intubation, laboratory parameters, therapy history, bacterial biology of sputum, types of tracheostomy and ventilator withdrawal. Comorbidities consisted of hypertension, diabetes, cardiac or cerebrovascular disease, and malignancy. Therapy history referred to anticoagulant therapy and transfusion therapy. Laboratory parameters were collected on the basis of venous blood samples. Intervention referred to the type of tracheostomy and ventilator withdrawal conducted. Surgical complications associated with tracheostomy were also analyzed. The surgical complications contained death, postoperative bleeding, wound infection, subcutaneous emphysema, pneumothorax and mediastinal emphysema, respiratory arrest, cannula prolapse, and tracheoesophageal fistula. We defined the degree of severity of COVID-19 (severe vs nonsevere) at the time of admission using the American Thoracic Society guidelines for community-acquired pneumonia $^{10}$.

\subsection{Analysis}

For baseline data, mean and rang range for data that were not normally distributed. Categorical variables were expressed as counts and percentages.

\section{RESULTS}

Of 1361 patients with confirmed SARS-CoV-2 infection, 247 patients (18.1.0\%) had non severe infection and 1114 patients $(81.9 \%)$ had severe infection according to their respiratory status. Overall, 11 patients $(0.8 \%)$ with tracheostomy were included in the analysis. All of them were elective tracheostomy, including percutaneous tracheostomy which was preferred and conventional open tracheostomy for patients with difficult airway. Their demographic and clinical characteristics were shown in Table 1 . Their mean (rang) age was $66.2(32-93)$ years, and $7(63.6 \%)$ were male. Of these patients, $9(81.2 \%)$ had at least 1 of the following comorbidities: hypertension $(8[72.3 \%])$, diabetes $(2[18.2 \%])$, cardiac or cerebrovascular disease $(4[36.4 \%])$, and malignancy $(1[9.1 \%])$. Of these 11 patients with tracheostomy, 11 patients (100\%) had at least 1 of the following underlying disorders: Coagulation function abnormity (10 [90.9\%]), including D-dimer abnormity $(10[90.9 \%])$ and APTT abnormity $(4[36.4 \%])$, anaemia $(9[81.8 \%])$, thrombocytopenia $(5[45.5 \%])$; liver function abnormity $(5[45.5 \%])$; renal function abnormity $(4[36.4 \%])$.

All of these patients with tracheostomy were severe COVID-19 patients which previously received endotracheal intubation and mechanical ventilation. Before tracheostomy, the mean (range) hospitalization day was 
$26.6(6,58)$ days, and the mean (range) duration of intubation was $16.8(6,36)$ days. Only 1 patient with cerebral hemorrhage was performed percutaneous tracheostomy 6 days after the brain surgery and endotracheal intubation, while the other 10 patients were performed tracheostomy at least 12 days after endotracheal intubation. Of 11 patients with tracheostomy, 10 patients $(90.9 \%)$ received anticoagulant therapy and 6 patients $(54.5 \%)$ received transfusion therapy before tracheostomy, to correct the coagulation disorders. Of these patients, bacterial biology of sputum before tracheostomy demonstrated that 11 patients (100\%) had at least 1 infection of the following bacteria: Klebsiella pneumoniae (5 [45.5\%]), Acinetobacter baumannii (7 [63.6\%]), Pseudomonas aeruginosa $(4[36.4 \%])$, and Stenotrophomonas maltophilia (5 [45.5\%]).

Finally, after tracheostomy, 9 patients $(81.8 \%$ ) acquired ventilator withdrawal, and the mean (range) duration of intubation after tracheostomy was $6.9(2,19)$ days. In patients with percutaneous tracheostomy, the mean (range) duration of intubation between tracheostomy and ventilator withdrawal was $10.4(4,19)$ days. In patients with conventional open tracheostomy, the mean (range) duration of intubation between tracheostomy and ventilator withdrawal was $2.5(2,4)$ days.

Table 2 showed the surgical complications during the tracheostomy or in the postoperative period. There were no patients with any of the following complications: death, postoperative bleeding, pneumothorax and mediastinal emphysema, respiratory arrest, cannula prolapse, and tracheoesophageal fistula. Of these patients with tracheostomy, $2(18.2 \%)$ had wound infection, and $1(9.1 \%)$ had subcutaneous emphysema. Overall, according to our protocol for the management of tracheostomy (Figure 1), both percutaneous tracheostomy and conventional open tracheostomy were safe for patients, if health workers performed adequate preoperative evaluation and preparation, and good surgery operation.

Moreover, no healthcare workers associated with the tracheostomy are confirmed to be infected by SARS$\mathrm{CoV}-2$, after 14 days out of working environment. All the conventional open tracheostomy were performed by doctors from otorhinolaryngology (Figure $2 \mathrm{~A}$ and $2 \mathrm{~B}$ ) according to the method described in our previous paper ${ }^{11}$, and the percutaneous tracheostomy were performed by doctors from intensive care unit according to the standard method. Patients' care were performed by nurses from intensive care unit and otorhinolaryngology. In addition to personal protective equipment (PPE), heal and moisture exchanger (HME) united closed tracheal suction catheters were strongly recommended to use in care for patients with tracheostomy or endotracheal intubation (Figure $2 \mathrm{C}$ and $2 \mathrm{D}$ ), in order to avoid secretion splashing. According to our protocol of tracheostomy and nursing (Figure 3), all of the doctors and nurses, including anesthesiologists for tracheostomy and nurses in the operation room, are safe during the tracheostomy or in the postoperative period.

\section{DISCUSSION}

To our knowledge, this is the first report on the demographic and clinical characteristics of COVID-19 patients with tracheostomy in the epicenter of China. As of April 6, 2020, 1361 patients with confirmed SARS-CoV-2 infection were hospitalized in our hospital (West Branch), including 1114 patients (81.9\%) with severe infection according to their respiratory status, and 11 patients $(0.8 \%)$ with tracheostomy were included in the analysis. All of the tracheostomies were elective tracheostomy, including two types of tracheostomy: percutaneous tracheostomy ( 6 cases) and conventional open tracheostomy ( 5 cases). In tracheotomy and postoperative period, splashing of airway secretions caused by open airway-related wound, cough reflex and ventilator ventilation may lead to the direct splashing of virus-carrying secretions to medical personnel and massive formation of aerosol in surgical environment, thus greatly increasing the risk of nosocomial dissemination. Therefore, in addition to the safety of patients, the important issue of the tracheotomy is to protect the healthcare workers and the surgical environment to avoid nosocomial infection.

For patients with severe COVID-19, endotracheal intubation and mechanical ventilation are important treatments to maintain their vital signs. However, if prolonged endotracheal intubation can not effectively facilitate the discharge of sputum, and this situation may seriously affected the airway patency of the patient and ventilator withdrawal, and then tracheotomy should be considered. However, for elective tracheotomy, intensivists, anesthesiologists, and otolaryngologists should conduct a comprehensive pre-surgery assessment 
to select the appropriate point in time and type of tracheotomy. Whether early tracheotomy after mechanical ventilation with intubation could facilitate the early withdrawal of ventilator is still a controversial issue ${ }^{12-13}$. However, there is a consensus among all disciplines that the removal of endotracheal intubation can increase the comfort of patients, reduce respiratory dead space and work of breathing, and reduce the use of sedatives which is helpful for reviving patients and restoring the cough reflex ${ }^{13}$. Therefore, with comprehensive multidisciplinary assessment, elective tracheotomy may be generally considered when extubation can not be performed after more than 7 days of endotracheal intubation, or in a short period of time.

Usually, percutaneous tracheotomy is preferred8, which is a minimally invasive surgical method with the advantages of less time-consuming ${ }^{14}$, less splashing and being a bedside operation ${ }^{15}$. However, some patients are not suitable for percutaneous tracheotomy because of possible difficulties in exposure of cervical trachea (e.g. humpback, short neck, obesity, enlarged thyroid, neck cicatricial contracture, et al.). At this situation, conventional open tracheostomy is an inevitable choice. But many doctor may think it is much more dangerous than percutaneous tracheotomy, and possibly influence the choice of tracheostomy under the background of COVID-19 pandemic. However, according to our optimized protocol for conventional open tracheostomy ${ }^{11}$, this is about to be reversed. As shown in Figure 3, the operators performed conventional open tracheostomy under general anesthesia with application of muscle relaxants, in order to completely avoid the cough reflex of patients during surgery. And the anesthesiologists help to move the endotracheal tube (ETT) close to carina of the trachea and this action can make the ETT cuff deeper than the incision site to prevent the ETT cuff leak due to an accidental damage to the cuff when making the tracheal opening. And when making the tracheal opening, a brief pause for ventilator is conducted. In a word, it's all about constantly keeping the lung, trachea and ventilator as an enclosed environment before making incision on the cervical trachea. Therefore, the safety of healthcare workers can be achieved during conventional open tracheostomy.

For each candidate of tracheostomy, adequate preoperative evaluations were needed. First, it is essential to acquire the anesthesiologist assessment for possibility and safety of general anesthesia, especially in conventional open tracheostomy. In the condition of general anesthesia, the use of sedative anesthetic and muscle relaxant can effectively avoid the cough reflex during tracheotomy. Second, transfusion therapy for severe coagulation function abnormity, thrombocytopenia and anaemia, should be considered before tracheotomy. Of 11 patients with tracheostomy, 10 patients (90.9\%) recieved anticoagulant therapy because of high DDimer levels and 6 patients (54.5\%) recieved transfusion therapy before tracheostomy. Although we can not get their coagulation function to the normal level, it is still necessary to ameliorate the situation for the safety of COVID-19 patients with elective tracheostomy. Of these 11 patients with tracheostomy, we tried to adjust APTT prolonged time less than 10 seconds, hemoglobin $(\mathrm{Hg})$ content not less than $60 \mathrm{~g} / \mathrm{L}$, and platelet (PLT) count not less than $60 \times 10^{12} / \mathrm{L}$. Of course, these are not absolute restrictions. In one case with APTT prolonged time more than 20 seconds, the patient received conventional open tracheostomy, and no surgical complications occurred, with much more careful manipulation of operators. Third, it is also important to take into consideration the impacts of ventilator associated pneumonia (acterial biology of sputum and the duration of endotracheal intubation, et al.). Of 11 patients with tracheostomy, the mean (range) duration of intubation before tracheostomy was $16.8(6,36)$ days, and bacterial biology of sputum before tracheostomy demonstrated that 11 patients $(100 \%)$ had at least one of pathogenic bacteria of ventilator associated pneumonia. In this situation, tracheostomy should be considered to be effective and helpful for the recovery of respiratory diseases.

According to our protocol, comprehensive evaluation and preparation before tracheostomy, optimized procedures during tracheostomy, and special care after tracheostomy were utilized. No severe complications occurred during tracheostomy or postoperative period, and 9 cases $(81.8 \%)$ were evacuated from the ventilator after tracheostomy. According to our protocol of tracheostomy and nursing, no healthcare workers associated with the tracheostomy are confirmed to be infected by SARS-CoV-2. These findings suggested that it was safe and effective to perform tracheostomy in COVID-19 patients with long-term endotracheal intubation and mechanical ventilation, if necessary. 
In patients with conventional open tracheostomy, the mean (range) duration of intubation between tracheostomy and ventilator withdrawal was $2.5(2,4)$ days, which was much shorter than that of percutaneous tracheostomy $(10.4[4,19]$ days). These results may be due to the fact that in the early stage of the outbreak of COVID-19, the patients were often in more severe condition and percutaneous tracheostomy were preferred, because of concerns about for the safety of healthcare workers and the stress on medical resources. These severe patients with percutaneous tracheostomy often needed much more time for recovery. In late stage of the outbreak of COVID-19, more patients with difficult airway were considered for conventional open tracheostomy, since more medical resources can be acquired. Moreover, in the late stage the outbreak of COVID-19, more patients were also in recovery of the disease. Therefore, this was not due to the type of tracheostomy.

\section{CONCLUSION}

This study may offer important new clinical information on COVID-19 patients with tracheostomy. It is especially meaningful to learn that for those patients with severe COVID-19, tracheostomy could be safe and beneficial for the patients. Comprehensive evaluation before tracheostomy and optimized procedures during tracheostomy were necessary and useful. Moreover, during the epidemic period of COVID-19, both adequate personal protective equipment (PPE) and appropriate protocols of tracheostomy and nursing are important for the safety of healthcare workers. Therefore, these protocols should be a good choice for all surgeons performing the tracheostomy for Covid-19 patients. They are helpful to rescue severe patients, and at the same time, to protect healthcare workers from the threat of coronavirus infection.

\section{ACKNOWLEDGMENTS}

None.

\section{REFERENCES}

1. Zhu N, Zhang D, Wang W, et al. A Novel Coronavirus from Patients with Pneumonia in China, 2019. N Engl J Med. 2020;382(8):727-733.

2. Amodio E, Vitale F, Cimino L, et al. Outbreak of Novel Coronavirus (SARS-Cov-2): First Evidences From International Scientific Literature and Pending Questions. Healthcare (Basel). 2020;8(1).

3. Dilcher M, Werno A, Jennings LC. SARS-CoV-2: a novel deadly virus in a globalised world. N Z Med J. 2020;133(1510):6-11.

4. Lai CC, Shih TP, Ko WC, et al. Severe acute respiratory syndrome coronavirus 2 (SARS-CoV-2) and coronavirus disease-2019 (COVID-19): The epidemic and the challenges. Int J Antimicrob Agents. 2020;55(3):105924.

5. Li T. Diagnosis and clinical management of severe acute respiratory syndrome Coronavirus 2 (SARSCoV-2) infection: an operational recommendation of Peking Union Medical College Hospital (V2.0). Emerg Microbes Infect. 2020;9(1):582-585.

6. Lombardi A, Bozzi G, Mangioni D, et al. Duration of quarantine in hospitalized patients with severe acute respiratory syndrome coronavirus 2 (SARS-CoV-2) infection: a question needing an answer. J Hosp Infect. 2020.

7. Marraro GA, Spada C. Consideration of the respiratory support strategy of severe acute respiratory failure caused by SARS-CoV-2 infection in children. Zhongguo Dang Dai Er Ke Za Zhi. 2020;22(3):183-194.

8. Mehta C, Mehta Y. Percutaneous tracheostomy. Ann Card Anaesth. 2017;20(Supplement):S19-S25. 
9. van Doremalen N, Bushmaker T, Morris DH, et al. Aerosol and Surface Stability of SARS-CoV-2 as Compared with SARS-CoV-1. N Engl J Med. 2020;382(16):1564-1567.

10. Metlay JP, Waterer GW, Long AC, et al. Diagnosis and treatment of adults with community-acquired pneumonia: an official clinical practice guideline of the American Thoracic Society and Infectious Disease Society of America. Am J Respir Crit Care Med. 2019;200(7):e45-e67. doi:10. 1164/rccm.201908-1581ST.

11. Xiao H, Zhong Y, Zhang X, et al. How to avoid nosocomial spread during Tracheostomy for Covid-19 patients. Head Neck. 2020. https://doi.org/10.1002/hed.26167

12. Szakmany T, Russell P, Wilkes AR, et al. Effect of early tracheostomy on resource utilization and clinical outcomes in critically ill patients: meta-analysis of randomized controlled trials. $\mathrm{Br} \mathrm{J}$ Anaesth. 2015;114(3):396-405.

13. Arabi Y, Haddad S, Shirawi N, et al. Early tracheostomy in intensive care trauma patients improves resource utilization: a cohort study and literature review. Crit Care. 2004;8(5):R347-352.

14. Putensen C, Theuerkauf N, Guenther U, et al. Percutaneous and surgical tracheostomy in critically ill adult patients: a meta-analysis. Crit Care. 2014;18(6):544.

15. Miles BA, Schiff B, Ganly I, et al. Tracheostomy during COV-SARS-CoV-2 pandemic: Recommendations from the New York Head and Neck Society. Head Neck. 2020.

\section{Figure Legends}

Figure 1 The protocol for preoperative evaluations and choices of tracheostomy in COVID-19 patients. 


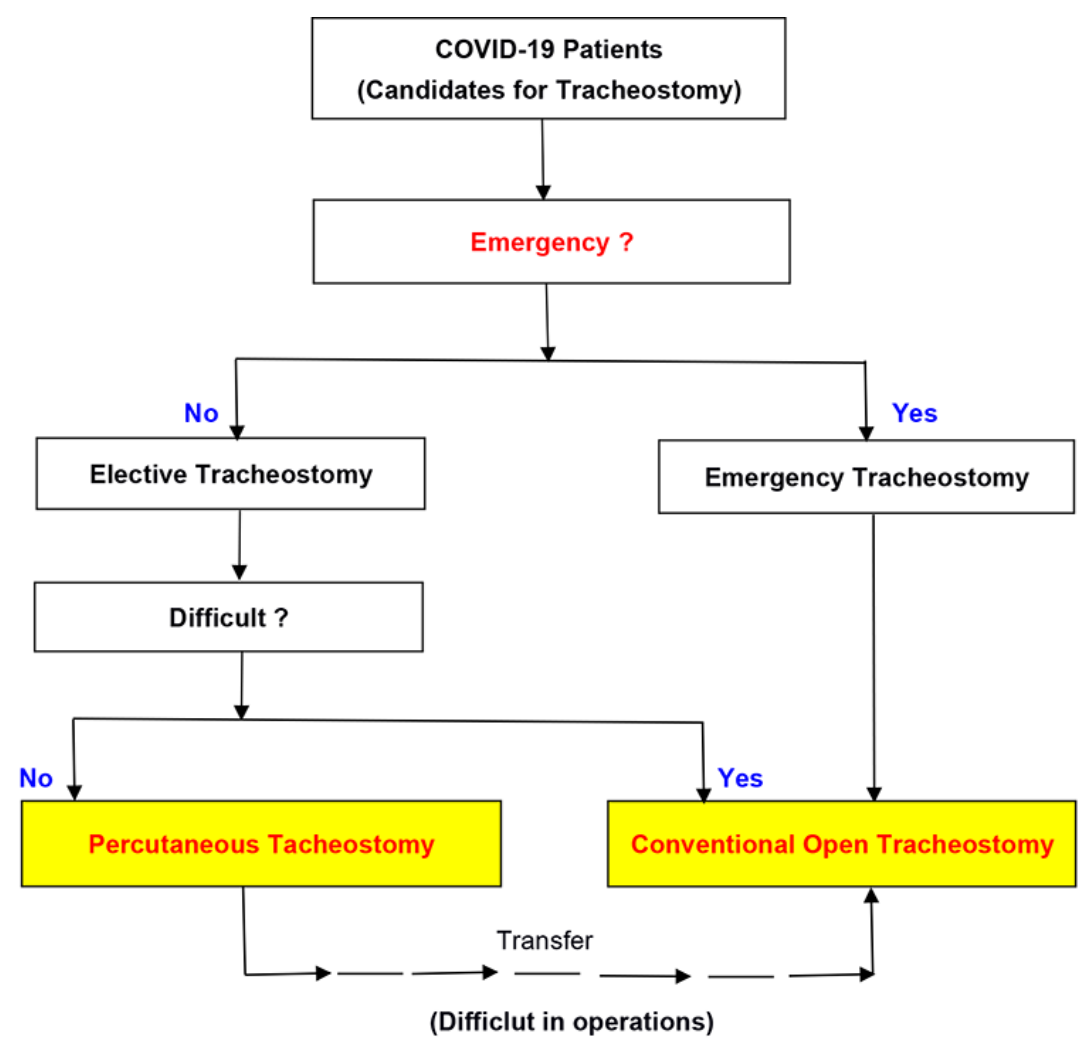

\section{Preoperative Evaluations:}

1. Anesthesiologist assessment for possiblity and safety of general anesthesia

2. Transfusion therapy for severe coagulation function abnormity, thrombocytopenia and anaemia

3. Possible difficulties in exposure of cervical trachea (e.g. humpback, short neck, obesity, enlarged thyroid, neck cicatricial contracture, et al.)

4. Impacts of ventilator associated pneumonia (acterial biology of sputum and the duration of endotracheal intubation, et al.)

\section{Aims:}

1.Reduce the use of sedatives 2.Recover cough reflex

Figure 1: This is a caption

Figure 2 The medical management and protection of of healthcare workers associated with the tracheostomy.

(A) The surgery team from otorhinolaryngology was performing conventional open tracheostomy.

(B) The personal protective equipment (PPE) for one member from the surgery team.

(C) Closed tracheal suction catheters were used in care for COVID-19 patients.

(D) The nurse was performing airway nursing for COVID-19 patients. 


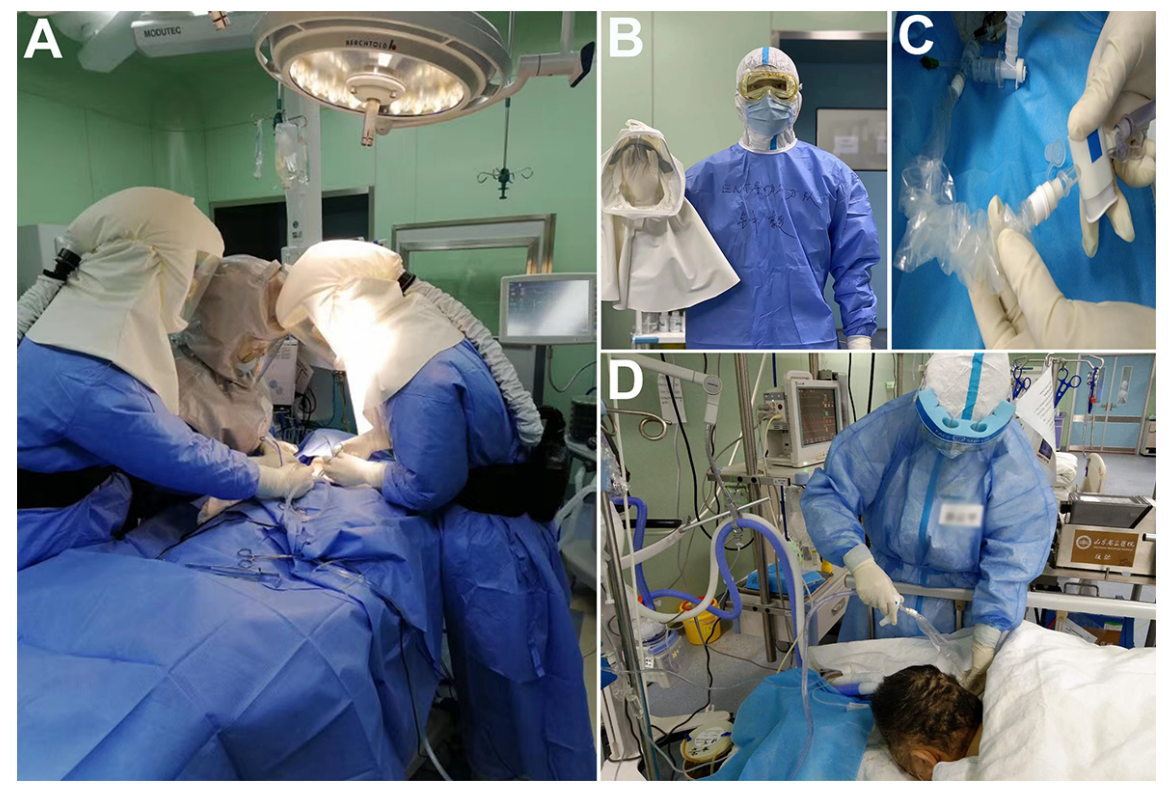

Figure 2: This is a caption

Figure 3 The three shields of healthcare workers in tracheostomy and nursing for COVID-19 patients. 


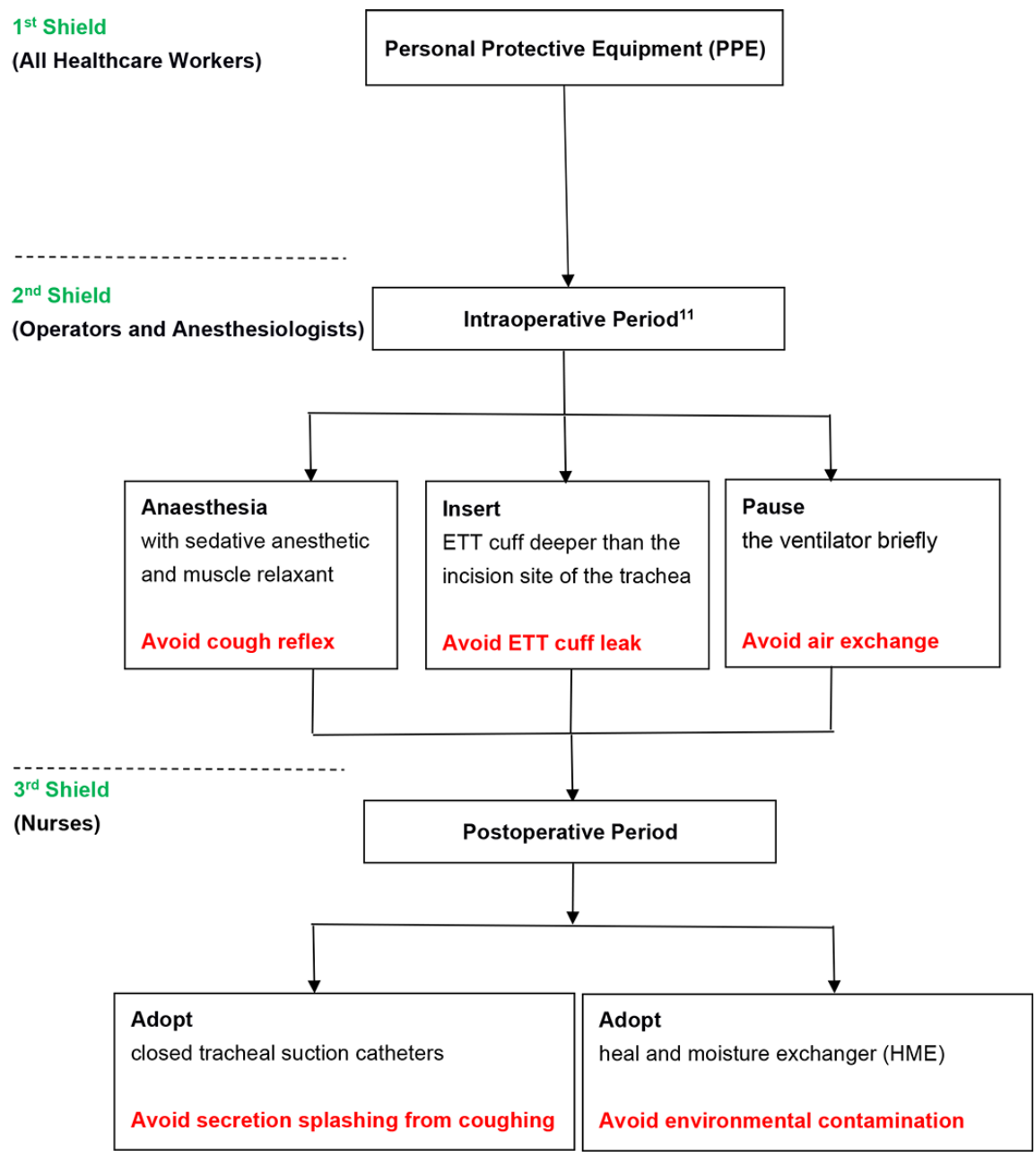

Figure 3: This is a caption

\section{Hosted file}

Table_1 (5).docx available at https://authorea.com/users/312328/articles/448168-safe-andeffective-management-of-tracheostomy-in-covid-19-patients

\section{Hosted file}

Table_2 (2).docx available at https://authorea.com/users/312328/articles/448168-safe-andeffective-management-of-tracheostomy-in-covid-19-patients 